\title{
Outcome of adjuvant radiotherapy after total hysterectomy in patients with uterine leiomyosarcoma or carcinosarcoma: a SEER- based study
}

Yuan $\mathrm{Li}^{1+}$, Haitao Ren ${ }^{2^{*}}$ and Junmei Wang ${ }^{1}$

\begin{abstract}
Background: The clinical impact of adjuvant radiotherapy on uterine sarcoma is unclear, and may depend on the histological type. Hence, the aim of this study was to evaluate clinical outcomes of adjuvant radiotherapy after total hysterectomy in patients with leiomyosarcoma or carcinosarcoma.

Methods: Data were obtained from the Surveillance, Epidemiology, and End Results (SEER) program. Cox proportional hazards regression analyses were performed to identify risk factors for overall mortality and cancerspecific mortality. In addition, a 1:1 propensity score matching approach was performed, in which age group, disease stage, tumor grade, tumor size, and lymphadenectomy status were matched.

Results: A total of 566 leiomyosarcoma and 1069 carcinosarcoma patients with stage I-III disease were included. Both regular Cox regression analysis and propensity score matching analysis revealed that utilization of adjuvant radiotherapy did not affect overall and cancer-specific mortality in patients with leiomyosarcoma. In contrast, for patients with carcinosarcoma, total mortality risk was significantly decreased with EBRT, brachytherapy, and combination radiotherapy compared with no radiotherapy. Cancer-specific mortality risk was significantly decreased with brachytherapy and combination radiotherapy as compared with no radiotherapy. Propensity score matching analyses revealed similar results in overall mortality, but not cancer-specific mortality, in patients with carcinosarcoma. Furthermore, the frequency of patients who did not receive any form of adjuvant radiotherapy was four times higher than those underwent adjuvant radiotherapy.
\end{abstract}

Conclusions: Adjuvant radiotherapy may provide a survival benefit for uterine carcinosarcoma, but not leiomyosarcoma. In addition, adjuvant radiotherapy is underutilized, and increased utilization of adjuvant radiotherapy may improve the survival rate of patients with carcinosarcoma.

Keywords: Adjuvant radiotherapy, Carcinosarcoma, Leiomyosarcoma, SEER, Uterine sarcoma

\section{Background}

Uterine sarcomas are rare malignant tumors arose from the smooth muscle or connective tissue of the uterus, which account for only 3 to $7 \%$ of uterine malignancies [1] but are the most common cause of uterine cancerspecific death [2]. Uterine sarcomas are histologically

\footnotetext{
* Correspondence: rht@zju.edu.cn

†Yuan Li and Haitao Ren contributed equally to this work

${ }^{2}$ Department of Burns and Wound Care Center, the Second Affiliated

Hospital, School of Medicine, Zhejiang University, Hangzhou 310009, China

Full list of author information is available at the end of the article
}

heterogeneous; two major histological subtypes are uterine leiomyosarcomas and carcinosarcomas that account for 30 and $50 \%$ of uterine sarcomas, respectively [1]. Leiomyosarcomas are smooth muscle tumors, expressing desmin, h-caldesmon, and smooth muscle $\alpha$ actin [1]. In contrast, carcinosarcomas are biphasic neoplasms consisting of admixed epithelial and mesenchymal elements, but are mostly of monoclonal origin [1, 3]. According to conversion theory, such biphasic tumors are likely as a result of late divergence and metaplasia of the carcinomatous component into the sarcomatous components

(C) The Author(s). 2019 Open Access This article is distributed under the terms of the Creative Commons Attribution 4.0 International License (http://creativecommons.org/licenses/by/4.0/), which permits unrestricted use, distribution, and 
$[3,4]$. Leiomyosarcomas often occurred in women aged 40 or older, and carcinosarcomas occurred in women who were much older [1].

Both uterine leiomyosarcomas and carcinosarcomas are highly aggressive with poor prognosis. Because of hematogenous spread, uterine leiomyosarcomas metastasize early primarily to the lungs [5]. In addition, at the time of diagnosis, one third of carcinosarcoma cases have already spread beyond the uterus [3]. Recurrence rate of leiomyosarcomas ranges from 53 to $71 \%$ [1], while recurrence rate of carcinosarcomas is $64 \%$ [3]. The 5-year overall survival (OS) rate was 15 to 20 and $30 \%$ for leiomyosarcomas and carcinosarcoma, respectively [6-8].

The primary curative treatment for uterine sarcomas is total abdominal hysterectomy, while debulking is used for palliative purpose if there is tumor outside of the uterus $[5,6]$. Removal of the ovaries and lymph node may be performed if disease is present; however, removal in all cases is debatable [6-8]. Survival in the presence of lymph node metastasis is significantly lower than if there is no metastasis to the lymph nodes; however, it is unclear if lymphadenectomy offers survival benefits [710].

The effect of adjuvant radiotherapy on uterine sarcoma is unclear, and may depend on the histological type $[6,11,12]$. Several retrospective studies have suggested that postoperative radiation decreases the local recurrence rate, but does not improve OS in patients with uterine sarcoma [6, 11-13]. In contrast, some studies have indicated that adjuvant radiotherapy does provide survival benefits for patients with leiomyosarcoma or carcinosarcoma [9, 11, 12, 14]. Furthermore, using competing risk modeling, Wong et al. (2013) demonstrated that adjuvant radiotherapy may improve both local recurrence rate and OS in patients with leiomyosarcoma [15]. The phase III EORTC trial that evaluated efficacy of adjuvant radiotherapy in patients with stage III uterine sarcomas found that adjuvant radiotherapy reduces any local recurrence rate by 37.5 and $48.9 \%$ in patients with leiomyosarcoma and carcinosarcoma, respectively [16].

To examine the hypothesis that the histological type of uterine sarcomas influences the effect of adjuvant postoperative radiotherapy on mortality in patients with uterine sarcoma, a population-based study was conducted, focusing on two major histological types, uterine leiomyosarcomas and carcinosarcomas. Thus, the present study aimed to evaluate the effect of adjuvant radiotherapy after total hysterectomy on overall mortality and cancer-specific mortality in patients with leiomyosarcoma or carcinosarcoma using data from the US National Cancer Institute (NCI) Surveillance, Epidemiology, and End Results (SEER) program database.

\section{Methods}

Data source

Data for this study were extracted from the US NCI SEER database that was released in April 2016, based on the November 2015 submission [17]. The SEER Data are collected from population-based registries, which cover approximately $28 \%$ of the US population. The SEER database contains information on patients' demographics, cancer incidence, primary tumor site, tumor morphology, stage at diagnosis, treatment, and follow-up status. The database is also linked to information on Medicare enrolment and Medicare claims, along with healthcare utilization and cost information for beneficiaries with cancer in the US.

Because all SEER data are de-identified, this study does not require Institutional Review Board approval, or informed consent by the study subjects. While, we obtained permission to access the SEER program data from the US National Cancer Institute (reference number: 11770-Nov2016).

\section{Study population}

The SEER database was examined to identify patients with primary uterine sarcoma according to codes of the International Classification of Diseases for Oncology $(\mathrm{ICD}-\mathrm{O})$ for anatomic sites (PRIMSITE $=$ C54.0-C54.3, C54.8-C54.9, C55.9) who underwent total hysterectomy (SURGPRIF $=40,50)$. Of them, patients with stage IV or unknown stage were excluded. Of the uterine sarcoma patients identified, patients with two major histological subtypes, leiomyosarcoma (HISTO3V $=8890,8891$, 8896) and carcinosarcoma (HISTO3V $=8980,8981$ ), were selected for the final analysis, because these 2 histological subtypes account for more than $75 \%$ of uterine sarcoma cases $[1,6]$. The SEER coding system classifies leiomyosarcoma into 4 grades: grade 1, well differentiated; grade 2, moderately differentiated; grade 3 , poorly differentiated; and undifferentiated/anaplastic.

\section{Data extraction}

Data obtained from the SEER database included patients' age at diagnosis, race, year of diagnosis, American Joint Committee on Cancer (AJCC) stage and grade, tumor size, treatments, and survival status (alive, death due to sarcoma, death due to other causes). Regarding adjuvant postoperative radiotherapy, patients were classified into four groups: i) no postoperative radiotherapy (RAD SURG $=0$ or RADIATN $=0$ ); ii) external beam radiation therapy (EBRT) alone (RAD_SURG $=3$ and RADIATN = 1 ); iii) brachytherapy (radioactive implants or radioisotopes) alone (RAD_SURG $=3$ and RADIATN $=2$ or 3 ); iv) combination radiotherapy (combination of EBRT and either radioactive implants or radioisotopes; RAD_ SURG $=3$ and RADIATN =4). Patients were also 
categorized according to lymphadenectomy status: yes, no, or unknown. Coding details and rules followed guidelines established by the SEER program (https:// seer.cancer.gov).

\section{Statistical analysis}

Categorical data were presented as count and percentage. Univariate and multivariate Cox proportional hazards regressions were performed to identify potential risk factors for overall mortality and cancer-specific mortality in patients with leiomyosarcoma or carcinosarcoma. Significant variables found in the univariate analysis $(P<0.05)$ were input into the multivariate Cox proportional hazards regression analysis using the stepwise selection method. To better balance patients who did and did not get radiation, a 1:1 propensity score matching approach was performed for an additional analysis, in which age group, disease stage, tumor grade, tumor size, and lymphadenectomy status were matched.

For overall mortality analysis, any cause of death was treated as an event, and survivors were treated as censors. For cancer-specific mortality analysis, deaths due to uterine sarcoma were events, and deaths from other causes or survivors were censors. Data were presented as adjusted hazard ratio (aHR) and 95\% confidence interval (CI) and $P$-value. To identify trends of adjuvant radiotherapy usage, the frequencies of adjuvant radiotherapy usage during the study period were counted. The significant level was set to 0.05 . Statistical analyses were performed by SAS statistical software (version 9.4, SAS Inc., Cary, NC, USA) and R software (version 3.2.2).

\section{Results}

\section{Study population}

A total of 4331 patients, who were diagnosed with uterine leiomyosarcoma or carcinosarcoma and underwent total hysterectomy, were identified in the SEER database during the period from 2004 to 2013. After excluding patients with AJCC stage IV or missing stage, 1635 patients were included in the final analysis.

\section{Patients' characteristics}

Patients' characteristics are summarized in Table 1. Of the 1635 included patients, 1028 (62.9\%) were over 60 years of age. Most of the patients were white (71.1\%), had stage I disease (65.4\%), and had a tumor size $>50$ $\mathrm{mm}(59.6 \%)$. The most frequently used adjuvant radiotherapy was EBRT that was applied to $14.8 \%$ of patients, followed by brachytherapy (9.7\%) and combination radiotherapy (5.8\%). However, $68.3 \%$ of the total population did not receive any form of adjuvant radiotherapy (Table 1).

Among 1635 eligible patients, 566 patients had leiomyosarcoma and 1069 had carcinosarcoma (Table 1). A large proportion of patients with leiomyosarcoma had undifferentiated/anaplastic tumors (25.8\%), and did not undergo a lymphadenectomy (66.1\%). In contrast, most patients with carcinosarcoma had grade 3 tumors (41.9\%), and $78.6 \%$ of patients underwent lymphadenectomy (Table 2).

The trend of adjuvant radiotherapy usage during the study period is shown in Fig. 1. Since patients with stage IV or unknown stage were excluded, the number of eligible patients in 2004-2009 was zero. As a result, only the period of 2010-2013 was shown in Fig. 1. During 2010-2013, the frequency of patients who did not receive any form of adjuvant radiotherapy was four times higher than those underwent adjuvant radiotherapy (Fig. $1)$.

During the study period, a total of 478 patients died. Of these 325 deaths were caused by uterine sarcoma, while others were not specified in the SEER database. The median survival time for the total population was 15 months (interquartile range [IQR]: 7-27 months). During the study period, 141 patients with leiomyosarcoma died, and only 111 out of 141 deaths were caused by leiomyosarcoma. The median survival time of all leiomyosarcoma patients was 16.5 months (IQR: 8-28 months). In contrast, 337 patients with carcinosarcoma died, and only 214 out of 337 deaths were caused by carcinosarcoma. The median survival time of all carcinosarcoma patients was 15 months (IQR: 6-26 months).

\section{Cox proportional hazards regression for overall and cancer-specific mortality}

Cox proportional hazards regression analyses were then performed to identify risk factors for overall and cancerspecific mortality in patients with leiomyosarcoma or carcinosarcoma. The results of the univariate Cox proportional hazards regression analyses of the associations of overall and cancer-specific mortality with adjuvant radiotherapy, demographic and clinical factors in patients with leiomyosarcoma or carcinosarcoma are shown in Additional file 1: Table S1.

Multivariate analyses revealed that for patients with leiomyosarcoma, there was no significant difference in overall and cancer-specific mortality with the use of any type of adjuvant radiotherapy as compared with no radiotherapy (Table 2). In contrast, for patients with carcinosarcoma, the risk of overall mortality was significantly decreased with EBRT alone (aHR $=0.72,95 \% \mathrm{CI}$ : $0.53,0.99, P=0.042)$, brachytherapy alone $(\mathrm{aHR}=0.55$, 95\% CI: $0.37,0.80, P=0.002)$, and combination radiotherapy $(\mathrm{aHR}=0.47,95 \% \mathrm{CI}: 0.29,0.77, P=0.003)$ as compared with no radiotherapy (Table 2). In addition, for patients with carcinosarcoma, the risk of cancerspecific mortality was significantly decreased with brachytherapy alone $(\mathrm{aHR}=0.51,95 \% \mathrm{CI}: 0.31,0.84, P=0.009)$, 
Table 1 Patients' characteristics

\begin{tabular}{|c|c|c|c|}
\hline & All & Leiomyosarcoma & $\begin{array}{l}\text { Carcinosarcoma } \\
n=1069\end{array}$ \\
\hline & $N=1635$ & $n=566$ & $n=1069$ \\
\hline Age $\geq 60$ years & $1028(62.9)$ & $175(30.9)$ & $1934(79.8)$ \\
\hline \multicolumn{4}{|l|}{ Race } \\
\hline White & $1162(71.1)$ & $397(70.1)$ & $765(71.6)$ \\
\hline Black & $324(19.8)$ & $111(19.4)$ & $214(20.0)$ \\
\hline Other $^{a}$ & $145(8.9)$ & $56(9.9)$ & $89(8.3)$ \\
\hline Unknown & $4(0.2)$ & $3(0.5)$ & $1(0.1)$ \\
\hline Year of diagnosis after 2009 & $1635(100)$ & $566(100.0)$ & $1069(100.0)$ \\
\hline \multicolumn{4}{|l|}{ AJCC Stage } \\
\hline Stage I & $1070(65.4)$ & $443(78.3)$ & $627(58.7)$ \\
\hline Stage ॥ & $153(9.4)$ & $66(11.7)$ & $87(8.1)$ \\
\hline Stage III & $412(25.2)$ & $57(10.0)$ & $355(33.2)$ \\
\hline \multicolumn{4}{|l|}{ Grade } \\
\hline Grade 1 & $34(2.1)$ & $21(3.7)$ & $13(1.2)$ \\
\hline Grade 2 & $81(5.0)$ & $53(9.3)$ & $28(2.6)$ \\
\hline Grade 3 & $539(33.0)$ & $91(16.1)$ & $448(41.9)$ \\
\hline Undifferentiated/anaplastic & $380(23.2)$ & $146(25.8)$ & $234(21.9)$ \\
\hline Unknown & $601(36.7)$ & $255(45.1)$ & $346(32.4)$ \\
\hline \multicolumn{4}{|l|}{ Tumor size } \\
\hline$\leq 50 \mathrm{~mm}$ & $461(28.2)$ & $87(15.4)$ & $374(35.0)$ \\
\hline$>50 \mathrm{~mm}$ & $974(59.6)$ & $424(74.9)$ & $550(51.5)$ \\
\hline Unknown & $200(12.2)$ & $55(9.7)$ & $145(13.5)$ \\
\hline \multicolumn{4}{|l|}{ Lymphadenectomy } \\
\hline No & $583(35.7)$ & $374(66.1)$ & $209(19.6)$ \\
\hline Yes & $1025(62.7)$ & $185(32.7)$ & 840 (78.6) \\
\hline Unknown & $27(1.7)$ & $7(1.2)$ & $20(1.8)$ \\
\hline \multicolumn{4}{|l|}{ Adjuvant radiation } \\
\hline No treatment & $1117(68.3)$ & $476(84.1)$ & $641(60.0)$ \\
\hline EBRT alone & $242(14.8)$ & $64(11.3)$ & $178(16.7)$ \\
\hline Brachytherapy alone & $159(9.7)$ & $11(1.9)$ & $148(13.8)$ \\
\hline Combination radiotherapy ${ }^{b}$ & $95(5.8)$ & $13(2.3)$ & $82(7.7)$ \\
\hline Others & $22(1.4)$ & $2(0.4)$ & $20(1.8)$ \\
\hline
\end{tabular}

AJCC American Joint Committee on Cancer, EBRT external beam radiation therapy Data are presented as number (percentage)

ancluding American Indian, Alaska native, Asian Pacific Islander, and other unspecified

${ }^{\mathrm{b} C o m b i n a t i o n}$ radiotherapy is EBRT with radioactive implants

combination radiotherapy $(\mathrm{aHR}=0.53,95 \% \mathrm{CI}: 0.29,0.95$, $P=0.034$ ) as compared to no radiotherapy (Table 2).

In addition, for patients with leiomyosarcoma, older age and higher stage were significantly associated with elevated risk of overall mortality, while higher stage (stage II and III) and larger tumor size were significantly associated with increased risk of cancer-specific mortality (all $p \leq 0.022$, Table 2). For patients with carcinosarcoma, higher age, stage III, and larger tumor size increased risk for overall mortality, but lymphadenectomy decreased risk of overall mortality (all $p \leq 0.001$, Table 2). Furthermore, stage III and larger tumor size elevated risk of cancer-specific mortality, whereas lymphadenectomy lower risk of cancer-specific mortality (all $p \leq 0.003$, Table 2).

\section{Overall and cancer-specific mortality in 1:1 propensity score matching sample}

To better balance the patients who did and did not receive radiotherapy, the associations between mortality and adjuvant radiotherapy type were also assessed by 
Table 2 Multivariate Cox proportional hazards regression analyses for overall and cancer-specific mortality in patients with leiomyosarcoma or carcinosarcoma

\begin{tabular}{|c|c|c|c|c|c|c|c|c|}
\hline \multirow[b]{3}{*}{ Variables } & \multicolumn{4}{|l|}{ Leiomyosarcoma } & \multicolumn{4}{|l|}{ Carcinosarcoma } \\
\hline & \multicolumn{2}{|l|}{ Overall $^{a}$} & \multicolumn{2}{|l|}{ Cancer-specific $^{b}$} & \multicolumn{2}{|l|}{ Overall $^{c}$} & \multicolumn{2}{|l|}{ Cancer-specific $^{d}$} \\
\hline & aHR $(95 \% \mathrm{Cl})$ & $P$-value & $\operatorname{aHR}(95 \% \mathrm{Cl})$ & $P$-value & aHR $(95 \% \mathrm{Cl})$ & $P$-value & $\operatorname{aHR}(95 \% \mathrm{Cl})$ & $P$-value \\
\hline \multicolumn{9}{|l|}{ Adjuvant radiotherapy } \\
\hline No treatment & reference & & reference & & reference & & reference & \\
\hline EBRT alone & $0.90(0.54,1.51)$ & 0.697 & $0.91(0.51,1.64)$ & 0.760 & $0.72(0.53,0.99)$ & 0.042 & $0.78(0.54,1.14)$ & 0.199 \\
\hline Brachytherapy alone & $0.57(0.14,2.31)$ & 0.429 & $0.47(0.07,3.38)$ & 0.452 & $0.55(0.37,0.80)$ & 0.002 & $0.51(0.31,0.84)$ & 0.009 \\
\hline Combination radiotherapy ${ }^{e}$ & NA & NA & NA & NA & $0.47(0.29,0.77)$ & 0.003 & $0.53(0.29,0.95)$ & 0.034 \\
\hline \multicolumn{9}{|l|}{ Age } \\
\hline$<60$ years & reference & & & & reference & & & \\
\hline$\geq 60$ years & $1.55(1.11,2.18)$ & 0.011 & & & $1.74(1.27,2.37)$ & 0.001 & & \\
\hline \multicolumn{9}{|l|}{ AJCC Stage } \\
\hline Stage I & reference & & reference & & reference & & reference & \\
\hline Stage ॥ & $1.82(1.15,2.89)$ & 0.011 & $1.85(1.09,3.12)$ & 0.022 & $1.06(0.67,1.69)$ & 0.802 & $1.10(0.61,1.98)$ & 0.758 \\
\hline Stage III & $3.07(2.01,4.69)$ & $<0.001$ & $3.21(2.02,5.13)$ & $<0.001$ & $2.36(1.88,2.97)$ & $<0.001$ & $2.57(1.93,3.42)$ & $<0.001$ \\
\hline \multicolumn{9}{|l|}{ Grade } \\
\hline Grade 1 & reference & & & & & & & \\
\hline Grade 2 & $1.58(0.18,13.60)$ & 0.679 & & & & & & \\
\hline Grade 3 & $7.17(0.97,52.86)$ & 0.053 & & & & & & \\
\hline Undifferentiated/anaplastic & $5.08(0.69,37.09)$ & 0.110 & & & & & & \\
\hline \multicolumn{9}{|l|}{ Tumor size } \\
\hline$\leq 50 \mathrm{~mm}$ & & & reference & & reference & & reference & \\
\hline$>50 \mathrm{~mm}$ & & & $2.98(1.38,6.45)$ & 0.006 & $1.96(1.50,2.57)$ & $<0.001$ & $2.07(1.46,2.93)$ & $<0.001$ \\
\hline \multicolumn{9}{|l|}{ Lymphadenectomy } \\
\hline No & & & & & reference & & reference & \\
\hline Yes & & & & & $0.61(0.47,0.78)$ & $<0.001$ & $0.61(0.45,0.84)$ & 0.003 \\
\hline
\end{tabular}

aHR adjusted hazard ratio, $\mathrm{Cl}$ confidence interval, EBRT external beam radiation therapy, AJCC American Joint Committee on Cancer ${ }^{a}$ Model was adjusted by age group, and stage, grade

${ }^{\mathrm{b}}$ Model was adjusted by stage, and tumor size

'Model was adjusted by age group, stage, tumor size, and lymphadenectomy

${ }^{\mathrm{d}}$ Model was adjusted stage, tumor size, and lymphadenectomy

e Combination radiotherapy: combination of EBRT and either radioactive implants or radioisotopes

NA, not available because no patient died during the study period

Unknown data for adjuvant radiotherapy, AJCC stage, grade, tumor size, and lymphadenectomy status were not shown in the table

Bold text indicates a significant difference with a $p$-value less than 0.05 compared to the reference group

Cox regression model in the 1:1 propensity score matching sample. Propensity score matching results indicated that for patients with leiomyosarcoma there was no significant difference in overall and cancer-specific mortality with the use of any type of adjuvant radiotherapy as compared with no radiotherapy (Table 3). For patients with carcinosarcoma, propensity score matching showed the risk of overall mortality was significantly decreased with EBRT alone $(\mathrm{aHR}=0.65,95 \% \mathrm{CI}: 0.45,0.93, P=$ $0.020)$, brachytherapy alone ( $\mathrm{aHR}=0.62,95 \% \mathrm{CI}: 0.40$, $0.95, P=0.029)$, and combination radiotherapy $(\mathrm{aHR}=$ $0.47,95 \% \mathrm{CI}: 0.26,0.85, P=0.013$ ) as compared with no radiotherapy (Table 3). However, no significant difference in cancer-specific mortality with the use of any type of adjuvant radiotherapy as compared with no radiotherapy in patients with carcinosarcoma was observed (Table 3).

Furthermore, non cancer-specific survival was analyzed and the results were presented in Additional file 1: Tables S2 and S3 (comparison between patients with and without adjuvant radiotherapy). For patients with leiomyosarcoma, their non cancer-specific survivals were not significantly different between those with or without adjuvant radiotherapy (Additional file 1: Table S3). However, the significant difference in non cancer-specific survival between those with or without adjuvant radiotherapy was observed in patients with carcinosarcoma (Additional file 1: Tables S2 and S3). 


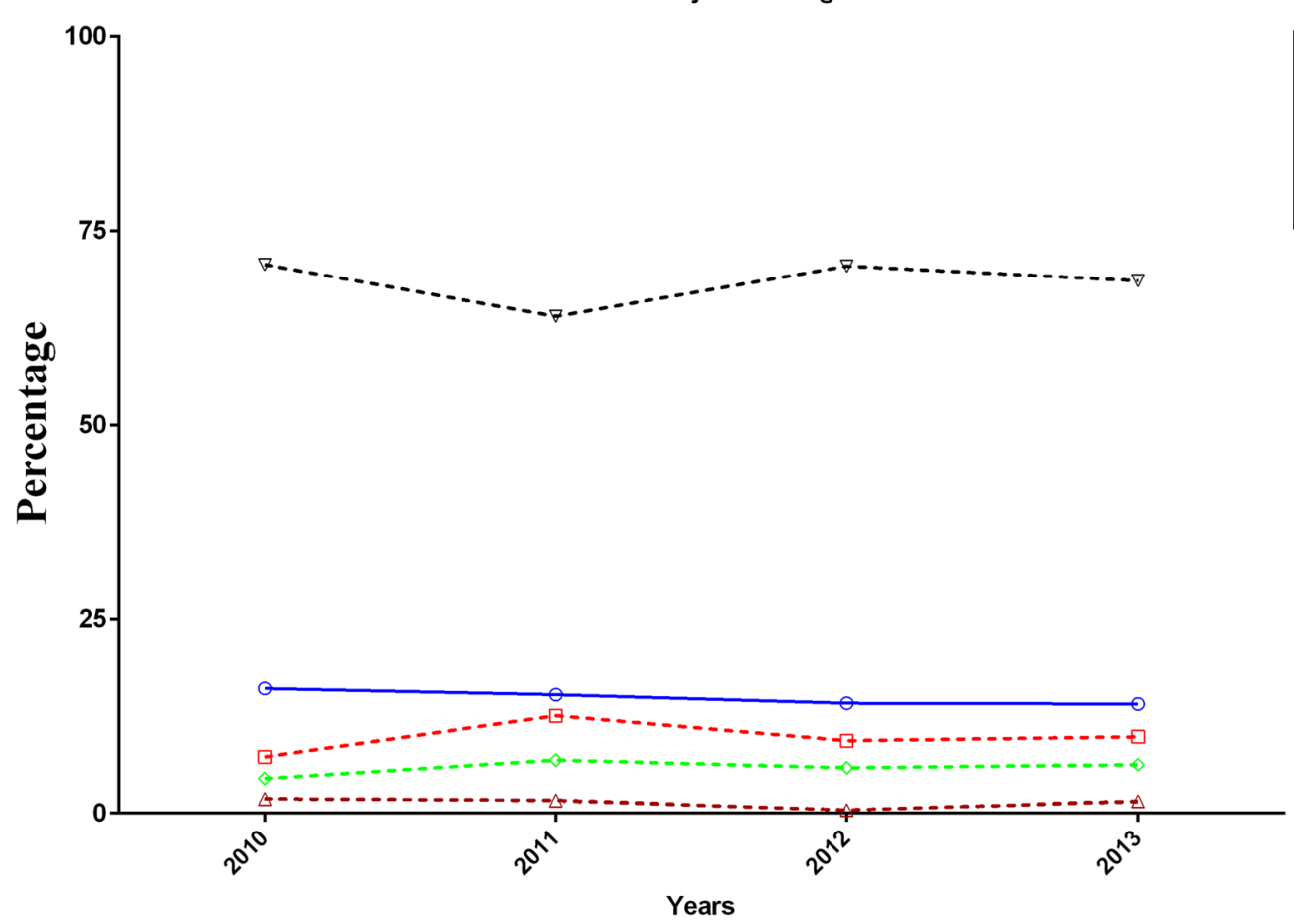

Fig. 1 Trend of adjuvant radiation usage. Utilization of adjuvant radiation per year was presented in percentage

\section{Discussion}

The present study used the US NCI SEER database to determine the effect of adjuvant radiotherapy on survival of patients with uterine leiomyosarcoma or carcinosarcoma. The results of the standard Cox proportional regression analyses showed that there was no difference in overall mortality or cancer-specific mortality in patients with leiomyosarcoma whether or not any type of radiotherapy was used. Whereas in patients with carcinosarcoma overall mortality risk was significantly decreased with EBRT alone, brachytherapy alone, and combination radiotherapy as compared to no radiotherapy, and the risk of cancer-specific mortality was significantly decreased with brachytherapy alone and combination radiotherapy as compared to no radiotherapy. The results of propensity score matching analyses revealed that no association between mortality and the use of adjuvant radiotherapy in patients with leiomyosarcoma. In contrast, EBRT alone, brachytherapy alone, and combination radiotherapy significantly lowered the risk of overall mortality, but not cancer-specific mortality, in patients with carcinosarcoma. The results of the present study

Table 3 Cox proportional hazards regression for overall and cancer-specific mortality of leiomyosarcoma and carcinosarcoma in the 1:1 propensity score matching sample

\begin{tabular}{|c|c|c|c|c|c|c|c|c|}
\hline \multirow[b]{3}{*}{ Variables } & \multicolumn{4}{|c|}{ Leiomyosarcoma $(n=180)$} & \multicolumn{4}{|c|}{ Carcinosarcoma $(n=856)$} \\
\hline & \multicolumn{2}{|l|}{ Overall $^{b}$} & \multicolumn{2}{|l|}{ Cancer-specific $^{c}$} & \multicolumn{2}{|l|}{ Overall $^{b}$} & \multicolumn{2}{|l|}{ Cancer-specific $^{c}$} \\
\hline & $\mathrm{HR}(95 \% \mathrm{Cl})$ & $P$-value & aHR (95\% Cl) & $P$-value & $\mathrm{HR}(95 \% \mathrm{Cl})$ & $P$-value & $\mathrm{aHR}(95 \% \mathrm{Cl})$ & $P$-value \\
\hline \multicolumn{9}{|l|}{ Adjuvant radiotherapy } \\
\hline No treatment & reference & & reference & & reference & & reference & \\
\hline EBRT alone & $0.71(0.33,1.54)$ & 0.386 & $0.69(0.27,1.79)$ & 0.447 & $0.65(0.45,0.93)$ & 0.020 & $0.78(0.50,1.22)$ & 0.274 \\
\hline Brachytherapy alone & $0.68(0.14,3.30)$ & 0.633 & $0.45(0.05,3.93)$ & 0.469 & $0.62(0.40,0.95)$ & 0.029 & $0.60(0.34,1.05)$ & 0.074 \\
\hline Combination radiotherapy ${ }^{a}$ & NA & NA & NA & NA & $0.47(0.26,0.85)$ & 0.013 & $0.67(0.33,1.33)$ & 0.250 \\
\hline
\end{tabular}

aHR adjusted hazard ratio, $\mathrm{Cl}$ confidence interval, EBRT external beam radiation therapy

${ }^{a}$ Combination radiotherapy: combination of EBRT and either radioactive implants or radioisotopes

bUnivariate regression model

${ }^{C}$ Race was adjusted for in multivariate regression model

NA: not available because no patient died during the study period

Unknown data for adjuvant radiotherapy were not shown in the table

Bold text indicates a significant difference with a $p$-value less than 0.05 compared to the reference group 
suggest that adjuvant radiotherapy might be underutilized, and proper use of adjuvant radiotherapy after surgery might improve survival of patients with uterine carcinosarcoma.

Uterine sarcomas are uncommon, highly aggressive tumors, while definitive guidelines for their management have yet to be determined. This is especially true with respect to adjuvant therapy with studies providing varying results as to its effectiveness [2, 18]. By analyzing SEER database (2002-2012), Hosh et al. reported that among 13,089 cases of uterine sarcoma, carcinosarcoma (53\%) was the most commonly diagnosed subtype followed by leiomyosarcoma (24\%) and stromal sarcoma (14\%), and patients with leiomyosarcoma and stromal sarcoma had significantly lower overall mortality than those with carcinosarcoma $(\mathrm{HR}=0.88,95 \%$ CI: $0.83-$ 0.94 , and $\mathrm{HR}=0.67,95 \% \mathrm{CI}: 0.67-0.73$, respectively) [6]. In addition, the overall mortality was significantly better in patients who had surgery with radiation than those who had surgery alone $(\mathrm{HR}=0.89,95 \% \mathrm{CI}: 0.83,0.95)$ [6]. Compared to the study of Hosh et al. [6], the present SEER-based study analyzed SEER database (2004-2013), estimated both overall morality and cancer-specific mortality, and focused on leiomyosarcoma or carcinosarcoma only but not all subtypes of uterine sarcoma. Regarding adjuvant radiotherapy, Hosh et al. [6] focused on the use of EBRT, whereas the present study included EBRT, brachytherapy, and combination radiotherapy. Furthermore, the present study performed propensity score matching analyses, so the corresponding results representing better balance the patients who did and did not receive adjuvant radiotherapy.

Sampath et al. [12] performed a retrospective analysis of 3650 patients with uterine sarcoma who were identified in the Impac Medical Systems (Sunnyvale, CA, USA) National Oncology Database, and found that in the whole cohort adjuvant radiotherapy was not predictive of OS, whereas in patients who did not have metastasis but underwent definitive surgery, adjuvant radiotherapy was associated with longer locoregional failure-free survival. Sampath et al. [12] defined adjuvant radiotherapy as postoperative EBRT with or without brachytherapy. Overall, the authors concluded that the use of adjuvant radiotherapy reduced the risk of locoregional failure at 5 years by $53 \%$ [12]. Cha et al. [19] examined the use of radiotherapy in 235 patients with primary uterine carcinosarcoma, of which $41 \%$ received adjuvant radiotherapy. The locoregional failure rate was lower for patients who received adjuvant radiotherapy than for those who did not $(17.5 \%$ vs. $28.5 \%, P=0.107)$, and adjuvant radiotherapy was associated with longer locoregional recurrence-free survival in patients who did not undergo pelvic lymph node dissection $(52.7 \%$ vs. $18.7 \%, P<0.001$ ) [19]. Moreover, a study of 141 stage I-
III uterine sarcoma patients demonstrated that postoperative radiotherapy, composing both EBRT and brachytherapy, with a total dose higher than 50 Gy significantly reduced the local recurrence rate [20].

Our results showed no difference in overall mortality or cancer-specific mortality in patients with leiomyosarcoma regardless whether or not any type of radiotherapy was used. In contrast, the regular Cox regression analyses revealed that in patients with carcinosarcoma overall mortality and cancer-specific mortality were decreased with the use of adjuvant radiotherapy, although the significant differences in cancer-specific mortality were no longer present in the propensity score matching analyses. These findings are consistent with recent evidence that leiomyosarcoma and carcinosarcoma have different molecular characteristics, which may be responsible for their different susceptibility to radiation $[21,22]$.

Nemani et al. [13] analyzed the SEER data of 1855 patients with uterine carcinosarcoma and found that lymphadenectomy was associated with improved OS in patients with stage I-III disease as compared to no lymphadenectomy. However, they also reported that and adjuvant radiotherapy was not associated with any increase in survival [13]. On the contrary, Clayton Smith et al. [14] also used SEER data to analyze 2461 women with carcinosarcoma, of who 890 received adjuvant radiotherapy. For women who received adjuvant radiotherapy as compared to those who did not, the 5-year OS rates were 41.5 and $33.2 \%$, respectively $(P<0.001)$, and uterine-specific survival rates were 56.0 and $50.8 \%$, respectively $(P=0.005)$ [14]. Radiotherapy was associated with better OS in patients with stage I-III disease (HR = $0.87, P=0.03)$, and with better $\mathrm{OS}(\mathrm{HR}=0.63, P<0.001)$ and uterine-specific survival $(\mathrm{HR}=0.63, P=0.004)$ in patients with stage IV disease [14].

There is no adjuvant therapy that has been consistently shown to improve outcomes for patients with leiomyosarcoma, and as such there are no clear guidelines for treatment [23]. A retrospective review of 208 patients with leiomyosarcoma reported that adjuvant radiotherapy did not improve survival outcomes [9]. A study of SEER data that included approximately 3000 patients from 1988 to 2004 with stage I and II disease found that adjuvant radiotherapy had no effect on survival for early-stage leiomyosarcoma, but that adjuvant radiotherapy reduced the risk of death by $21 \%$ in women with carcinosarcoma [23]. In patients with carcinosarcoma, adjuvant radiotherapy substantially reduced mortality in women who did not undergo lymph node dissection, but in node-negative women [23]. Consistently, our results also demonstrated that use of adjuvant radiotherapy was associated with reduced overall and cancer-specific mortality in patients with carcinosarcoma. 
However, the opposite results have been also reported. Mahdavi et al. [11] reviewed 147 patients with leiomyosarcoma treated at 11 regional medical centers from 1985 to 2005 and found the 5-year survival of patients who received radiotherapy was significantly higher than those who did not ( $70 \%$ vs. $35 \%$ ); however, the survival advantage was no longer present at 7.5 years. In addition, the pelvic recurrence rate was lower in the radiotherapy group ( $18 \%$ vs. $49 \%, P=0.02$ ) [11].

This study has inherent strengths and limitations. The SEER data has been found to be generalizable to the US population [24], and as it is a high-quality national database discrepancies and potential biases are reduced. The SEER database, however, does not contain information of comorbidities, lifestyle and risk factors, environmental exposure, and family history, and thus these factors could not be examined. The SEER database also does not include data on quality of surgery (morcellation), margins, radiation dose and target location, cohort of patients treated prior to the Food \& Drug Administration (FDA) notice on uterine tumor morcellation, reliability of cancer specific survival, and cause of death reports. Furthermore, the SEER database also suffers several limitations, such as missing data on grade and stage, unreliable information on cause of death, and potential selection bias. Chemotherapy is also used for the adjuvant treatment of uterine sarcomas, and may improve survival [25]. However, the SEER database does not incorporate information on the use of chemotherapy. This could potentially affect the results as we could not include chemotherapy administration in the analysis. Furthermore, we did not examine outcomes with neoadjuvant radiotherapy. Outcome estimates are different when different staging systems are used (AJCC or International Federation of Gynecology and Obstetrics [FIGO]), and this was not examined [26, 27].

\section{Conclusions}

The results of this population-based study demonstrated that the clinical impact of adjuvant radiotherapy is histological type-specific, with significant decreased overall mortality rate in patients with uterine carcinosarcoma, but not uterine leiomyosarcoma. In addition, adjuvant radiotherapy was underutilized, and increased use of adjuvant radiotherapy might improve the survival rate of patients with carcinosarcoma.

\section{Additional file}

Additional file 1: Table S1. Univariate Cox proportional hazards regression for overall and cancer-specific mortality of leiomyosarcoma and carcinosarcoma. Table S2. Multivariate Cox proportional hazards regression for non cancer-specific mortality of leiomyosarcoma or carcinosarcoma in 1:1 propensity score matching sample. Table S3. Multivariate
Cox proportional hazards regression for non cancer-specific mortality of leiomyosarcoma or carcinosarcoma in 1:1 propensity score matching sample. (DOCX $22 \mathrm{~kb}$ )

\section{Abbreviations}

aHR: Adjusted hazard ratio; AJCC: American Joint Committee on Cancer; Cl: Confidence interval; EBRT: External beam radiation therapy; FDA: Food \& Drug Administration; FIGO: International Federation of Gynecology and Obstetrics; ICD-O: International Classification of Diseases for Oncology; IQR: Interquartile range; NCl: National Cancer Institute; OS: Overall survival; SEER: Surveillance, Epidemiology, and End Results

\section{Acknowledgements}

The authors acknowledge the efforts of the Surveillance, Epidemiology, and End Results (SEER) Program in the creation of the SEER database. The interpretation and reporting of these data are the sole responsibility of the authors.

\section{Authors' contributions}

$Y L, H R$, and JW developed the study. $Y L$ and HR analyzed the patient data. $Y L$ and $H R$ were major contributors in writing the manuscript. All authors discussed the results and approved the final manuscript.

\section{Funding}

This research was supported by Zhejiang Provincial Natural Science Foundation of China under Grant No. LY15H150004 and General Research Program of Zhejiang Provincial Department of Health under Grant No.201345919. The funders had no role in study design, data collection and analysis, decision to publish, or preparation of the manuscript.

\section{Availability of data and materials}

Data for this study were obtained from the US NCI SEER database (https:// seer.cancer.gov) that was released in April 2016, based on the November 2015 submission.

\section{Ethics approval and consent to participate}

Because patient data in the SEER database were de-identified, signed informed consent was waived for the present study.

\section{Consent for publication}

Not applicable.

\section{Competing interests}

The authors declare that they have no competing interests.

\section{Author details}

${ }^{1}$ Department of Ultrasound, Women's Hospital, School of Medicine, Zhejiang University, Hangzhou 310006, China. ${ }^{2}$ Department of Burns and Wound Care Center, the Second Affiliated Hospital, School of Medicine, Zhejiang

University, Hangzhou 310009, China.

Received: 16 August 2018 Accepted: 25 June 2019

Published online: 15 July 2019

\section{References}

1. Prat J, Mbatani N. Uterine sarcomas. Int J Gynaecol Obstet. 2015;131(Suppl 2):S105-10. https://doi.org/10.1016/j.ijgo.2015.06.006.

2. Chern JY, Boyd LR, Blank SV. Uterine sarcomas: the latest approaches for these rare but potentially deadly tumors. Oncology. 2017;31:229-36.

3. Cherniack AD, Shen $H$, Walter $V$, Stewart C, Murray BA, Bowlby $R$, et al. Integrated molecular characterization of uterine Carcinosarcoma. Cancer Cell. 2017;31:411-23. https://doi.org/10.1016/j.ccell.2017.02.010.

4. Singh R. Review literature on uterine carcinosarcoma. J Cancer Res Ther. 2014;10:461-8.

5. Reichardt P. The treatment of uterine sarcomas. Ann Oncol. 2012;23(Suppl 10):x151-7.

6. Hosh M, Antar S, Nazzal A, Warda M, Gibreel A, Refky B. Uterine sarcoma: analysis of 13,089 cases based on surveillance, epidemiology, and end results database. Int J Gynecol Cancer. 2016;26:1098-104. https://doi.org/10. 1097/IGC.0000000000000720. 
7. Kapp DS, Shin JY, Chan JK. Prognostic factors and survival in 1396 patients with uterine leiomyosarcomas: emphasis on impact of lymphadenectomy and oophorectomy. Cancer. 2008;112:820-30. https://doi.org/10.1002/cncr. 23245.

8. Seagle BL, Kanis M, Kocherginsky M, Strauss JB, Shahabi S. Stage I uterine carcinosarcoma: matched cohort analyses for lymphadenectomy, chemotherapy, and brachytherapy. Gynecol Oncol. 2017;145:71-7. https:// doi.org/10.1016/j.ygyno.2017.01.010.

9. Giuntoli RL 2nd, Metzinger DS, DiMarco CS, Cha SS, Sloan JA, Keeney GL. Retrospective review of 208 patients with leiomyosarcoma of the uterus: prognostic indicators, surgical management, and adjuvant therapy. Gynecol Oncol. 2003;89:460-9.

10. Gadducci A. Prognostic factors in uterine sarcoma. Best Pract Res Clin Obstet Gynaecol. 2011;25:783-95. https://doi.org/10.1016/j.bpobgyn.2011.06. 002.

11. Mahdavi A, Monk BJ, Ragazzo J, Hunter MI, Lentz SE, Vasilev SA, et al. Pelvic radiation improves local control after hysterectomy for uterine leiomyosarcoma: a 20-year experience. Int J Gynecol Cancer. 2009;19:10804. https://doi.org/10.1111/IGC.0b013e3181acae50.

12. Sampath S, Schultheiss TE, Ryu JK, Wong JY. The role of adjuvant radiation in uterine sarcomas. Int J Radiat Oncol Biol Phys. 2010;76:728-34. https://doi. org/10.1016/j.jijobp.2009.02.077.

13. Nemani D, Mitra N, Guo M, Lin L. Assessing the effects of lymphadenectomy and radiation therapy in patients with uterine carcinosarcoma: a SEER analysis. Gynecol Oncol. 2008;111:82-8. https:/doi. org/10.1016/j.ygyno.2008.05.016.

14. Clayton Smith D, Kenneth Macdonald O, Gaffney DK. The impact of adjuvant radiation therapy on survival in women with uterine carcinosarcoma. Radiother Oncol. 2008;88:227-32. https://doi.org/10.1016/j. radonc.2007.11.029.

15. Wong P, Han K, Sykes J, Catton C, Laframboise S, Fyles A, et al. Postoperative radiotherapy improves local control and survival in patients with uterine leiomyosarcoma. Radiat Oncol. 2013;8:128. https://doi.org/10. 1186/1748-717X-8-128

16. Reed NS, Mangioni C, Malmström H, Scarfone G, Poveda A, Pecorelli S, et al. Phase III randomised study to evaluate the role of adjuvant pelvic radiotherapy in the treatment of uterine sarcomas stages I and II: an European Organisation for Research and Treatment of Cancer Gynaecological Cancer group study (protocol 55874). Eur J Cancer. 2008;44: 808-18. https://doi.org/10.1016/j.ejca.2008.01.019.

17. National Cancer Institute. Surveillance, Epidemiology, and End Results Program (SEER). https://seer.cancer.gov/. Accessed 20 Feb 202019.

18. Strander H, Turesson I, Cavallin-Ståhl E. A systematic overview of radiation therapy effects in soft tissue sarcomas. Acta Oncol. 2003;42:516-31.

19. Cha J, Kim YS, Park W, Kim HJ, Kim JY, Kim JH, et al. Clinical significance of radiotherapy in patients with primary uterine carcinosarcoma: a multicenter retrospective study (KROG 13-08). J Gynecol Oncol. 2016;27:e58. https://doi. org/10.3802/jgo.2016.27.e58.

20. Vitale SG, Laganà AS, Capriglione S, Angioli R, La Rosa VL, Lopez S, et al. Target therapies for uterine carcinosarcomas: current evidence and future perspectives. Int J Mol Sci. 2017;18. https://doi.org/10.3390/ijms18051100.

21. Davidson B, Micci F. Molecular characteristics of uterine sarcomas. Expert Rev Mol Diagn. 2017;17:515-22. https://doi.org/10.1080/14737159.2017. 1311790.

22. Matsuo K, Omatsu K, Ross MS, Johnson MS, Yunokawa M, Klobocista MM, et al. Impact of adjuvant therapy on recurrence patterns in stage I uterine carcinosarcoma. Gynecol Oncol. 2017;145:78-87. https://doi.org/10.1016/j. ygyno.2017.02.001

23. Wright JD, Seshan VE, Shah M, Schiff PB, Burke WM, Cohen CJ, et al. The role of radiation in improving survival for early-stage carcinosarcoma and leiomyosarcomas. Am J Obstet Gynecol. 2008;199:536.e1-8. https://doi.org/ 10.1016/j.ajog.2008.04.019 Epub 2008 Jun 3.

24. Kuo TM, Mobley LR. How generalizable are the SEER registries to the cancer populations of the USA? Cancer Causes Control. 2016;27(9):1117-26. https:// doi.org/10.1007/s10552-016-0790-x Epub 2016 Jul 21.

25. Terek MC, Akman L, Hursitoglu BS, Sanli UA, Ozsaran Z, Tekindal MA, et al. The retrospective analysis of patients with uterine sarcomas: a single-center experience. J Cancer Res Ther. 2016;12:309-13. https://doi.org/10.4103/09731482.148698.

26. Giuntoli RL 2nd, Lessard-Anderson CR, Gerardi MA, Kushnir CL, Cliby WA Metzinger DS, et al. Comparison of current staging systems and a novel staging system for uterine leiomyosarcoma. Int J Gynecol Cancer. 2013;23: 869-76. https://doi.org/10.1097/IGC.0b013e3182916a1e.

27. Zivanovic O, Leitao MM, lasonos A, Jacks LM, Zhou Q, Abu-Rustum NR, et al. Stage-specific outcomes of patients with uterine leiomyosarcoma: a comparison of the International Federation of Gynecology and Obstetrics and American joint committee on Cancer staging systems. J Clin Oncol. 2009;27:2066-72. https://doi.org/10.1200/JCO.2008.19.8366.

\section{Publisher's Note}

Springer Nature remains neutral with regard to jurisdictional claims in published maps and institutional affiliations.
Ready to submit your research? Choose BMC and benefit from:

- fast, convenient online submission

- thorough peer review by experienced researchers in your field

- rapid publication on acceptance

- support for research data, including large and complex data types

- gold Open Access which fosters wider collaboration and increased citations

- maximum visibility for your research: over $100 \mathrm{M}$ website views per year

At BMC, research is always in progress.

Learn more biomedcentral.com/submissions 